\title{
Generalized Boltzmann equations for on-shell particle production in a hot plasma
}

\author{
A. Jakovác* \\ Theory Division, CERN, CH-1211 Geneva 23, Switzerland
}

(Received 5 July 2002; published 11 December 2002)

\begin{abstract}
A novel refinement of the conventional treatment of Kadanoff-Baym equations is suggested. In addition to the Boltzmann equation, another differential equation is used for calculating the evolution of the nonequilibrium two-point function. Although it was usually interpreted as a constraint on the solution of the Boltzmann equation, we argue that its dynamics is relevant to the determination and resummation of the particle production cut contributions. The differential equation for this new contribution is illustrated in the example of the cubic scalar model. The analogue of the relaxation time approximation is suggested. It results in the shift of the threshold location and in a smearing out of the nonanalytic threshold behavior of the spectral function. The possible consequences for the dilepton production are discussed.
\end{abstract}

DOI: 10.1103/PhysRevD.66.125003

PACS number(s): 11.10.Wx, 05.30.- d, 24.60.-k, 25.75.Dw

\section{INTRODUCTION}

Real photon production and dilepton production in a hot plasma are intensively discussed subjects in the current literature. One aspect of this discussion is the behavior of the production rate near a zero invariant mass of the lepton pair. Numerical simulations [1] find a vanishing rate, while higher loop perturbative [2] and hard-thermal-loop- (HTL-) improved [3] calculations find diverging results in this limit. The possibility that the two-loop order enhancement may be a consequence of infrared (IR) singularities was studied in recent papers [4], where also an effective description was suggested to overcome the problem. In [5], a resummation method was worked out for ladder diagrams, and it was applied to the photon and gluon production rate.

Resummation in perturbation theory becomes unavoidable, in general, when we encounter IR divergences that enhance the contribution of higher order loops, thus overriding the coupling constant suppression. A well-known example is the on-mass-shell IR divergence of the propagator due to a radiative mass shift. This divergence is cured by resumming one-particle-irreducible (1PI) diagrams with the help of Schwinger-Dyson (SD) equations. This example also illustrates that resummation may be effective only for some specific momentum range, while in other regions its effect might remain negligible. From this point of view, also finitetemperature Debye screening is a consequence of IR divergences.

In nonequilibrium systems, there appear new sources of IR divergences. When working in real time, one recognizes that higher loops may give contributions that grow as some power of the elapsed time ( $\sim t^{\alpha n}$, where $n$ is the loop order). This would clearly restrict the applicability of perturbation theory to rather short times, so we need a resummation. It turns out [6] that resummation leads to Boltzmann equations describing the temporal evolution of the propagator. In Fourier space, the corresponding phenomenon is the appearance of pinch singularities $[7,8]$ in two-particle intermediate states with a vanishing momentum difference. The resummation of

\footnotetext{
*Email address: Antal.Jakovac@cern.ch
}

these divergences, which involves ladder diagrams, leads, again, to Boltzmann equations [9].

In this paper, we shall argue that there are further momentum ranges where we can observe pinch singularities. When these momentum ranges are relevant, we have to perform appropriate resummation also there. To see the origin of these divergences, we have to analyze the corresponding pinch singularities, as will be done in Sec. IV. To demonstrate here what happens, we mention that the core effect of pinch singularities is that in a pair of propagators one of them can force the other to its mass shell: in formulas there appears $\sim \varrho\left(p_{1}\right) G\left(p_{2}\right)$, which is singular for $p_{1}=p_{2}$. If we parametrize the momenta carried by the particles as $p_{1,2}$ $=Q \pm K / 2$, then we can find for the pinch singular term two, formally equivalent, expressions,

$$
\begin{aligned}
\frac{2 \delta\left((Q-K / 2)^{2}-m^{2}\right)}{(Q+K / 2)^{2}-m^{2}} & =\frac{\delta\left((Q-K / 2)^{2}-m^{2}\right)}{Q K} \\
& =\frac{\delta\left((Q-K / 2)^{2}-m^{2}\right)}{Q^{2}+K^{2} / 4-m^{2}} .
\end{aligned}
$$

The first equality suggests that divergences occur when $Q K$ $=0$ : then the resummation leads to the Boltzmann equation. The last form shows that, at least formally, we could equivalently use the $Q^{2}+K^{2} / 4-m^{2}=0$ condition.

The formal equivalence of the above formulas ends, however, when we consider Landau prescription, applied to $k_{0}$ [using $K=\left(k_{0}, \mathbf{k}\right), Q=\left(q_{0}, \mathbf{q}\right)$ notation]. In the first form, there appears $i \varepsilon\left(2 q_{0}+k_{0}\right)$ in the denominator, while the second and third form contain $i \varepsilon q_{0}$ and $i \varepsilon k_{0} / 2$, respectively. Since only the sign of the coefficient of $i \varepsilon$ is relevant, we can rewrite Eq. (1) as

$$
\begin{aligned}
\frac{2 \delta\left((Q-K / 2)^{2}-m^{2}\right)}{(Q+K / 2)^{2}-m^{2}}= & \delta\left((Q-K / 2)^{2}-m^{2}\right) \\
& \times\left(\frac{\Theta\left(\left|2 q_{0}\right|-\left|k_{0}\right|\right)}{Q K}\right. \\
& \left.+\frac{\Theta\left(\left|k_{0}\right|-\left|2 q_{0}\right|\right)}{Q^{2}+K^{2} / 4-m^{2}}\right),
\end{aligned}
$$


where the notation of Landau prescription was suppressed. This equation suggests that the Boltzmann equation should be valid only for $\left|2 q_{0}\right|>\left|k_{0}\right|$; if $\left|2 q_{0}\right|<\left|k_{0}\right|$, the first-order Boltzmann kernel $Q K$ has to be substituted by the secondorder kernel $Q^{2}+K^{2} / 4-m^{2}$. This is the new kinematical situation studied in the present paper.

After identifying the IR divergences, we have to design an appropriate resummation scheme for them. In order to perform ladder resummation, we can use the linearized Kadanoff-Baym (KB) equations [9]. These come from a procedure (for details see Secs. IIIC and IV) that consists of writing two SD equations for each of the Wigner-Fouriertransformed propagators, next taking their sum and difference, and finally linearizing them. They read, schematically,

$$
\begin{gathered}
2 Q K \bar{G}(Q, K)=\left(\mathcal{A}_{-} * \bar{G}\right)(Q, K), \\
2\left(Q^{2}+\frac{K^{2}}{4}-m^{2}\right) \bar{G}(Q, K)=\left(\mathcal{A}_{+} * \bar{G}\right)(Q, K),
\end{gathered}
$$

where $\mathcal{A}_{ \pm}$denote the linear operators acting on the propagators. The equations are diagonal in $K$, which is the momentum associated with the average coordinate of the propagator. The coefficient of $\bar{G}$ vanishes on the left-hand side in regions of pinch singularity, while the expression is finite on the right-hand side. The perturbative solution diverges at every order in any such regime, but the complete solution of the differential equations is regular. This is the resummation realized with the help of SD equations. Using other resummation techniques will give the same result, at least to leading order of the pinch-singular terms [9].

The complete solution has to satisfy both Eqs. (3), but these describe, due to Landau prescription, different kinematical regimes (for details, see Sec. III). Therefore, one of the equations can be satisfied only as a constraint. In the usual approach, one treats the difference equation $\left[\mathcal{A}_{-}\right.$in Eqs. (3)] as a differential equation (this is the quantum Boltzmann equation), while the sum equation $\left[\mathcal{A}_{+}\right.$in Eqs. (3) $]$is treated as a constraint. However, as was indicated above around Eq. (2), and will be further argued in Secs. III and IV, this description may be valid only for $\left|2 q_{0}\right|>\left|k_{0}\right|$; if $\left|2 q_{0}\right|$ $<\left|k_{0}\right|$, then we should use the sum equation as a differential equation and the difference equation as a constraint. In both of these cases, we arrive at valid, but different, solutions: for the general solution of the system of Eqs. (3) we have to add them up.

Computing expectation values of composite operators involves integration over $Q$, which is the momentum associated with the relative coordinate. After this integration, more insight is offered into the physics of the different contributions. At one loop order the discontinuity of the Boltzmann term $\sim 1 /(Q K)$ gives a nonzero result for $K^{2}<0$ - this is the Landau damping regime. The discontinuity of the other contribution $\sim 1 /\left(Q^{2}+K^{2} / 4-m^{2}\right)$ is nonzero when $K^{2}>4 m^{2}$, where $2 m$ is the threshold value for particle production-we call this a normal cut regime. The solution of the Boltzmann equation in the relaxation time approximation generates a finite imaginary part to $k_{0}$; this is why a quantity, conserved at tree level, can decay exponentially due to radiative effects.
In a kind of similar relaxation time approximation, higher loop pinch singularities provide imaginary and real contributions to the denominator $Q^{2}+K^{2} / 4-m^{2}$, which results in a shift of the threshold location and in a "smoothing" of the threshold singularity.

In this paper, the effects described above in qualitative terms will be demonstrated in the cubic scalar model. Beyond its simplicity it shows some similarity with fermiongauge systems as far as the perturbation theory is concerned. We choose a simple observable, $\left\langle\left[\varphi^{2}(x), \varphi^{2}(0)\right]\right\rangle$; this is the simplified analogue of $\left\langle\left[j_{\mu}(x), j^{\mu}(0)\right]\right\rangle$, which plays an important role in the theory of dilepton production [10]. In Sec. II, we introduce the model and our observable. In Sec. III, we perform a thorough one-loop calculation, identify the different kinematical regions, and examine the way SD equations and $\mathrm{KB}$ equations reproduce the one-loop result. In Sec. IV, we turn to higher loops. We show the origin of pinch singularities, and apply KB equations to resum these singularities in different kinematic regions. We also solve the corresponding equations in the relaxation time approximation. In Sec. V, we make some comments on the relevance of our results to the dilepton production in fermion-gauge systems. Section VI then closes the paper by presenting our conclusions and an outlook.

\section{THE MODEL}

The cubic scalar model has the Lagrangian

$$
\mathcal{L}=\frac{1}{2}(\partial \varphi)^{2}-\frac{1}{2} m^{2} \varphi^{2}-\frac{h}{6} \varphi^{3} .
$$

We are interested in the equilibrium expectation value of the composite spectral function,

$$
\left.\left\langle\left[\varphi^{2}(x), \varphi^{2}(0)\right]\right\rangle\right|_{1 \mathrm{PI},\langle\varphi\rangle=0} .
$$

This quantity corresponds to the expectation value $\left\langle\left[j_{\mu}(x), j^{\mu}(0)\right]\right\rangle$ in the fermion-gauge systems, which plays a central role in dilepton production [10]. The subscripts 1PI and $\langle\varphi\rangle=0$ will be omitted in the following. The quantity of phenomenological interest is the discontinuity of $i R(K)$, which is the Fourier transform of

$$
i R(x)=\Theta\left(x_{0}\right)\left\langle\left[\varphi^{2}(x), \varphi^{2}(0)\right]\right\rangle .
$$

$R(x)$, on the other hand, is the linear-response function of $\left\langle\varphi^{2}(x)\right\rangle$ to the mass modification of the Lagrangian characterized by the linear operator $\mathcal{P}$ :

$$
\begin{aligned}
\mathcal{L}_{\mathcal{P}} & =\mathcal{L}-\frac{1}{2} \mathcal{P} \varphi^{2} \\
& \Rightarrow \delta\left\langle\varphi^{2}\right\rangle(K)=\frac{1}{2} R(K) \mathcal{P}(K)+\cdots,
\end{aligned}
$$

where $\delta f=f-\left.f\right|_{\mathcal{P}=0}$ for any quantity. We will define $\left\langle\varphi^{2}(x)\right\rangle$ via point splitting; in the Keldysh and in the R/A formalisms (cf. Appendix A) we should compute 


$$
\begin{aligned}
\left\langle\varphi^{2}(x)\right\rangle & =\frac{1}{2} \lim _{y \rightarrow x}[\langle\varphi(x) \varphi(y)\rangle+\langle\varphi(y) \varphi(x)\rangle] \\
& =\frac{1}{2} \lim _{y \rightarrow x}\left[i G_{21}(x, y)+i G_{12}(x, y)\right] \\
& =\lim _{y \rightarrow x} i G_{r r}(x, y) .
\end{aligned}
$$

In Fourier space,

$$
\begin{aligned}
\left\langle\varphi^{2}\right\rangle(K)= & \int d x e^{i K x} \lim _{y \rightarrow x} \int d p_{1} d p_{2} \\
& \times e^{-i p_{1} x+i p_{2} y} i G_{r r}\left(p_{1}, p_{2}\right) \\
= & \int d Q i \bar{G}_{r r}(Q, K)
\end{aligned}
$$

$[\bar{G}(Q, K)$ denotes the Wigner-Fourier transform of $G(x, y)$, which has the usual two-variable Fourier-transform $G\left(p_{1}, p_{2}\right)$, cf. Appendix A]. We introduce the quantity $R(Q, K)$ defined through the relation

$$
\begin{aligned}
\delta i \bar{G}_{r r}(Q, K) & =\frac{1}{2} R(Q, K) \mathcal{P}(K) \\
& \Rightarrow R(K)=\int \mathrm{d} Q R(Q, K) .
\end{aligned}
$$

\section{ONE-LOOP PERTURBATION THEORY}

In leading order the only interaction we have to take into account is $-\mathcal{P} \varphi^{2} / 2$. In the Keldysh and R/A formalism (cf. Appendix A), this piece of the Lagrangian has the form

$$
-\frac{1}{2} \mathcal{P} \varphi^{2} \rightarrow-\frac{1}{2} \mathcal{P}\left(\varphi_{1}^{2}-\varphi_{2}^{2}\right)=-\mathcal{P} \varphi_{r} \varphi_{a}
$$

Linear response to $\mathcal{P}$ yields

$$
\begin{aligned}
\delta \bar{G}_{r r}(Q, K)= & {\left[\bar{G}_{r a}^{0}\left(Q+\frac{K}{2}\right) \bar{G}_{r r}^{0}\left(Q-\frac{K}{2}\right)\right.} \\
& \left.+\bar{G}_{a r}^{0}\left(Q-\frac{K}{2}\right) \bar{G}_{r r}^{0}\left(Q+\frac{K}{2}\right)\right] \mathcal{P}(K),
\end{aligned}
$$

where $G_{a b}^{0}$ means free equilibrium propagators. The coefficient of $\mathcal{P}$ identifies $-i R(Q, K) / 2$. Its discontinuity reads

$$
\begin{aligned}
\underset{k_{0}}{\operatorname{DisciR}(Q, K)=} & 2\left[\varrho^{0}\left(Q+\frac{K}{2}\right) i \bar{G}_{r r}^{0}\left(Q-\frac{K}{2}\right)\right. \\
& \left.-\varrho^{0}\left(Q-\frac{K}{2}\right) i \bar{G}_{r r}^{0}\left(Q+\frac{K}{2}\right)\right] .
\end{aligned}
$$

Using the notations

$$
\begin{gathered}
Q=\left(q_{0}, \mathbf{q}\right), \quad q=|\mathbf{q}|, \quad K=\left(k_{0}, \mathbf{k}\right), \\
k=|\mathbf{k}|, \quad x=\frac{\mathbf{q k}}{q k}, \quad \omega_{\mathbf{q}}^{2}=q^{2}+m^{2},
\end{gathered}
$$

the free equilibrium expressions read as

$$
\begin{gathered}
\varrho^{0}(Q)=2 \pi \operatorname{sgn}\left(q_{0}\right) \delta\left(q_{0}^{2}-\omega_{\mathbf{q}}^{2}\right), \\
i G_{r r}^{0}(Q)=2 \pi \operatorname{sgn}\left(q_{0}\right)\left(\frac{1}{2}+n\left(q_{0}\right)\right) \delta\left(q_{0}^{2}-\omega_{\mathbf{q}}^{2}\right) .
\end{gathered}
$$

Inserting them into Eq. (13), we find

$$
\begin{aligned}
\underset{k_{0}}{\operatorname{DisciR}(Q, K)=} & 8 \pi^{2} \operatorname{sgn}\left(q_{0}+\frac{k_{0}}{2}\right) \operatorname{sgn}\left(q_{0}-\frac{k_{0}}{2}\right) \\
& \times\left(n_{-}-n_{+}\right) \delta\left(\left(Q+\frac{K}{2}\right)^{2}-m^{2}\right) \\
& \times \delta\left(\left(Q-\frac{K}{2}\right)^{2}-m^{2}\right),
\end{aligned}
$$

where $n_{ \pm}=n\left(q_{0} \pm k_{0} / 2\right)$.

We first analyze the product of the $\delta$ functions (for details, see Appendix B). One can write them in various forms,

$$
\begin{aligned}
D & :=\delta\left(\left(Q+\frac{K}{2}\right)^{2}-m^{2}\right) \delta\left(\left(Q-\frac{K}{2}\right)^{2}-m^{2}\right) \\
& =\delta(2 Q K) \delta\left(Q^{2}+\frac{K^{2}}{4}-m^{2}\right) \\
& =\frac{1}{2 \mathcal{Z}\left|k_{0}^{2}-k^{2} x^{2}\right|} \delta\left(q_{0}-\frac{k x}{2} \operatorname{sgn}\left(k_{0}\right) \mathcal{Z}\right) \delta\left(q-\frac{\left|k_{0}\right|}{2} \mathcal{Z}\right),
\end{aligned}
$$

where $\mathcal{Z}=\sqrt{\left(K^{2}-4 m^{2}\right) /\left(k_{0}^{2}-k^{2} x^{2}\right)}$, which is real when $K^{2}>4 m^{2}$ or $K^{2}<0$ (see Appendix B).

The last form carries an important message concerning the relative magnitude of $q_{0}$ and $k_{0}$. For $K^{2}<0$, it requires that $\left|q_{0}\right|>\left|k_{0}\right| / 2$, while for $K^{2}>4 m^{2}$ we find $\left|q_{0}\right|<k / 2$ $<\left|k_{0}\right| / 2$ (see Appendix B). It has the consequence that in the present circumstances one can write

$$
\begin{aligned}
\operatorname{sgn}\left(q_{0} \pm \frac{k_{0}}{2}\right)= & \Theta\left(K^{2}-4 m^{2}\right)( \pm) \operatorname{sgn}\left(k_{0}\right) \\
& +\Theta\left(-K^{2}\right) \operatorname{sgn}\left(q_{0}\right),
\end{aligned}
$$

which implies, when inserting back into Eq. (16), that 


$$
\begin{aligned}
\underset{k_{0}}{\operatorname{DisciR}(Q, K)=} & \frac{4 \pi^{2}}{\sqrt{\left(k_{0}^{2}-k^{2} x^{2}\right)\left(K^{2}-4 m^{2}\right)}} \\
& \times \delta\left(q_{0}-\frac{k x}{2} \operatorname{sgn}\left(k_{0}\right) \mathcal{Z}\right) \delta\left(q-\frac{\left|k_{0}\right|}{2} \mathcal{Z}\right) \\
& \times\left\{\Theta ( K ^ { 2 } - 4 m ^ { 2 } ) \left[1+n\left(\frac{k_{0}}{2}-q_{0}\right)\right.\right. \\
& \left.+n\left(\frac{k_{0}}{2}+q_{0}\right)\right]+\Theta\left(-K^{2}\right)\left[n\left(q_{0}-\frac{k_{0}}{2}\right)\right. \\
& \left.\left.-n\left(q_{0}+\frac{k_{0}}{2}\right)\right]\right\}
\end{aligned}
$$

The $Q$ integration is performed using this form (see Appendix B), and one finds

$$
\begin{aligned}
\int \underset{k_{0}}{d Q \operatorname{DisciR}(Q, K)=} & \frac{\operatorname{sgn}\left(k_{0}\right)}{4 \pi k}\left\{\Theta\left(K^{2}-4 m^{2}\right) \int_{-k \mathcal{Z}_{1} / 2}^{k \mathcal{Z}_{1} / 2} d y\right. \\
& \times\left[1+n\left(\frac{\left|k_{0}\right|}{2}-y\right)+n\left(\frac{\left|k_{0}\right|}{2}+y\right)\right] \\
& +2 \Theta\left(-K^{2}\right) \int_{k \mathcal{Z}_{1} / 2}^{\infty} d y\left[n\left(y-\frac{\left|k_{0}\right|}{2}\right)\right. \\
& \left.\left.-n\left(y+\frac{\left|k_{0}\right|}{2}\right)\right]\right\}
\end{aligned}
$$

which agrees with the results of earlier calculations [11].

The limiting case usually investigated is that the spatial variation of the external source is small, i.e., $k \ll m, T$. Then, in the first term of Eq. (20), the variation of $y$ is of order $k$, thus $y \ll\left|k_{0}\right|$ and we can neglect the $y$ dependence of the integrand. In the second term $k \geqslant\left|k_{0}\right|$, so the small- $k$ approximation yields $\mathcal{Z}_{1} \approx 2 m /|K| \gg 1$. Then $y \gg k \geqslant k_{0}$ and we can perform an expansion in power series with respect to $k_{0}$. Finally, we obtain

$$
\begin{aligned}
\int \underset{k_{0}}{d Q \operatorname{DisciR}(Q, K) \approx} & \frac{1}{4 \pi}\left\{\Theta\left(K^{2}-4 m^{2}\right) \sqrt{1-\frac{4 m^{2}}{K^{2}}}\right. \\
& \times\left[1+2 n\left(\frac{k_{0}}{2}\right)\right] \\
& \left.+2 \Theta\left(-K^{2}\right) \frac{k_{0}}{k} n\left(\frac{k m}{|K|}\right)\right\}
\end{aligned}
$$

\section{A. The Landau damping region}

The approximate expression (21) can be derived without the detailed computation outlined above. If $K^{2}<0$ and $k$ $\ll m, T$, the plasma excitations behave as on-mass-shall particles because $Q^{2}=m^{2}-K^{2} / 4 \approx m^{2}$. At high temperatures all components of $Q$ are of the order of the temperature. In this regime, it is a good approximation to neglect $K$ in Eqs. (16) and (17) whenever it is possible, and to perform an expansion in power series with respect to the components of $K$ when it is necessary. This yields

$$
\begin{aligned}
\underset{k_{0}}{\operatorname{DisciR}(Q, K)} & \approx-8 \pi^{2} k_{0} \frac{d n\left(q_{0}\right)}{d q_{0}} \delta(2 Q K) \delta\left(Q^{2}-m^{2}\right) \\
& =-8 \pi^{2} k_{0} \frac{d n}{d \omega} \delta(2 Q K) \delta\left(Q^{2}-m^{2}\right),
\end{aligned}
$$

since $d n / d q_{0}$ is even in $q_{0}$. After the $Q$ integration, one readily reproduces the Landau damping piece of Eq. (21).

Although this is a straightforward consequence of our approximations, this result is still a bit surprising. The assumption we made is quite plausible: the variation of the background field $K$ is small as compared to the typical scale of the loop momentum, which is $T$ at high temperatures. This was implemented by requiring $Q \gg K$ for all components. This assumption, however, just reproduces the Landau cut region, while the normal cut is missing.

\section{B. The normal cut region}

Let us examine where we have lost the normal cut, and what are the appropriate assumptions that lead to its approximate form in Eq. (21), in a similarly direct way as the Landau cut is reproduced.

The answer is easy to find when we recall the earlier statement of the exact analysis that in the $K^{2}>4 m^{2}$ regime $\left|q_{0}\right|<k / 2<\left|k_{0}\right| / 2$ : that means that the assumption $Q \gg K$ is not valid for all components. In the normal cut regime, the timelike component of the loop momentum is small, even smaller than the spatial variation of the background field.

This observation suggests a complementary approximation scheme: we neglect the (spatial) $k$ dependence, not assuming, however, that $k_{0}<k$. In the product of the $\delta$ functions (17) we can then write

$$
\begin{aligned}
D & =\delta(2 Q K) \delta\left(Q^{2}+\frac{K^{2}}{4}-m^{2}\right) \\
& \rightarrow \delta\left(2 q_{0} k_{0}\right) \delta\left(Q^{2}+\frac{k_{0}^{2}}{4}-m^{2}\right) \\
& =\frac{1}{2\left|k_{0}\right|} \delta\left(q_{0}\right) \delta\left(\frac{k_{0}^{2}}{4}-m^{2}-q^{2}\right) .
\end{aligned}
$$

Therefore, in the leading order, we can assume $q_{0} \approx 0$ as well. We also see that we immediately obtain $k_{0}^{2}>4 m^{2}$ from the second $\delta$ function. The approximation for the discontinuity of $R$ then reads

$$
\begin{aligned}
\underset{k_{0}}{\operatorname{DisciR}(Q, K) \approx} & \frac{4 \pi^{2}}{\left|k_{0}\right|}\left[1+2 n\left(\frac{k_{0}}{2}\right)\right] \\
& \times \delta\left(q_{0}\right) \delta\left(\frac{k_{0}^{2}}{4}-m^{2}-q^{2}\right) .
\end{aligned}
$$


This expression reproduces, after $Q$ integration, the normal cut part of the approximated result (21).

It is interesting to observe that the approximations leading to Landau and normal cuts concern different factors of $D$ in Eq. (17): using $\delta\left(Q^{2}+K^{2} / 4-m^{2}\right)$ for small $K$ yields that $Q$ is on the mass shell, while using $\delta(2 Q K)$ for small $k$ yields $q_{0} \approx 0$. While both assumptions seem to be well justified, neither of them reproduces the complete discontinuity of $R$, just complementary parts of them. To understand this phenomenon better, we now turn to the Schwinger-Dyson equations.

\section{Schwinger-Dyson equations}

The general form of SD equations in the Keldysh and R/A formalisms can be found in Appendix A. At one-loop perturbation theory, we need the self-energies coming from the interaction Lagrangian (11):

$$
\Pi_{a r}(Q, K)=\Pi_{r a}(Q, K)=\mathcal{P}(K), \quad \Pi_{a a}=0,
$$

which has to be inserted into Eq. (A14) of Appendix A. For a linear response we insert free propagators into its righthand side. We are interested in the $r r$ component [cf. (9)],

$$
\left[\left(Q \pm \frac{K}{2}\right)^{2}-m^{2}\right] \bar{G}_{r r}(Q, K)=\mathcal{P}(K) \bar{G}_{r r}^{0}\left(Q \mp \frac{K}{2}\right) .
$$

First we show that the solution agrees with perturbation theory outlined above. The inverse of $(Q \pm K / 2)^{2}-m^{2}$ is the retarded or advanced Greens function, where we have to take into account that now we assign the $i \varepsilon$ term to $k_{0}$. Then the solutions of the two equations read

$$
\begin{aligned}
\bar{G}_{r r}(Q, K)= & \mathcal{P}(K) \bar{G}_{r a}^{0}\left(Q+\frac{K}{2}\right) \bar{G}_{r r}^{0}\left(Q-\frac{K}{2}\right) \\
& +C \delta\left(\left(Q+\frac{K}{2}\right)^{2}-m^{2}\right) \\
\bar{G}_{r r}(Q, K)= & \mathcal{P}(K) \bar{G}_{a r}^{0}\left(Q-\frac{K}{2}\right) \bar{G}_{r r}^{0}\left(Q+\frac{K}{2}\right) \\
& +C^{\prime} \delta\left(\left(Q-\frac{K}{2}\right)^{2}-m^{2}\right),
\end{aligned}
$$

where $C$ and $C^{\prime}$ are integration constants. The two solutions can be matched using the fact that $\bar{G}_{r r}^{0}(Q) \sim \delta\left(Q^{2}-m^{2}\right)$; as a result we obtain exactly Eq. (12).

Sometimes it may be more convenient to solve, instead of the original form, the sum and difference equations:

$$
\begin{aligned}
& \left(Q^{2}+\frac{K^{2}}{4}-m^{2}\right) \bar{G}_{r r}(Q, K) \\
& =\mathcal{P}(K) \frac{\bar{G}_{r r}^{0}\left(Q+\frac{K}{2}\right)+\bar{G}_{r r}^{0}\left(Q-\frac{K}{2}\right)}{2}
\end{aligned}
$$

$$
2 Q K \bar{G}_{r r}(Q, K)=\mathcal{P}(K)\left[\bar{G}_{r r}^{0}\left(Q-\frac{K}{2}\right)-\bar{G}_{r r}^{0}\left(Q+\frac{K}{2}\right)\right]
$$

The solution of the first equation satisfies the second one and vice versa; to see this, we recall that $\bar{G}_{r r}^{0}(Q) \sim \delta\left(Q^{2}-m^{2}\right)$, therefore

$$
\begin{aligned}
& 2 Q K\left[\bar{G}_{r r}^{0}\left(Q-\frac{K}{2}\right)+\bar{G}_{r r}^{0}\left(Q+\frac{K}{2}\right)\right] \\
& =\left(Q^{2}+\frac{K^{2}}{4}-m^{2}\right)\left[\bar{G}_{r r}^{0}\left(Q-\frac{K}{2}\right)-\bar{G}_{r r}^{0}\left(Q+\frac{K}{2}\right)\right] .
\end{aligned}
$$

The two solutions, on the other hand, are different when we apply Landau prescription (i.e., we treat the equations as differential equations with initial condition). Using Eq. (15) for the $r r$ propagator, the discontinuity of $i R(Q, K)$ coming from the solutions of Eqs. (28) and (29) reads

$$
\begin{aligned}
\underset{k_{0}}{\operatorname{DisciR}(Q, K)=} & 8 \pi^{2} \operatorname{sgn}\left(k_{0}\right)\left[\left(\frac{1}{2}+n_{+}\right) \operatorname{sgn}_{+}\right. \\
& \left.+\left(\frac{1}{2}+n_{-}\right) \operatorname{sgn}_{-}\right] \delta\left(Q^{2}+\frac{K^{2}}{4}-m^{2}\right) \\
& \times \delta(2 Q K), \\
\underset{k_{0}}{\operatorname{DisciR}(Q, K)=} & 8 \pi^{2} \operatorname{sgn}\left(q_{0}\right)\left[\left(\frac{1}{2}+n_{-}\right) \operatorname{sgn}_{-}\right. \\
& \left.-\left(\frac{1}{2}+n_{+}\right) \operatorname{sgn}_{+}\right] \delta\left(Q^{2}+\frac{K^{2}}{4}-m^{2}\right) \\
& \times \delta(2 Q K),
\end{aligned}
$$

respectively, where $\operatorname{sgn}_{ \pm}=\operatorname{sgn}\left(q_{0} \pm k_{0} / 2\right)$ and $n_{ \pm}=n\left(q_{0}\right.$ $\pm k_{0} / 2$ ). Comparing with the original solution (19), we can observe that the first form corresponds to the normal cut regime, if $\left|k_{0}\right| / 2>\left|q_{0}\right|$ (which also means, using the $\delta$ functions, that $K^{2}>4 m^{2}$ ), while it does not correspond to any solution if $\left|k_{0}\right| / 2<\left|q_{0}\right|$. For the second form one can state that for $\left|k_{0}\right| / 2<\left|q_{0}\right|$ (i.e., when $K^{2}<0$ ) it corresponds to the Landau-cut regime, while it is fake for $\left|k_{0}\right| / 2>\left|q_{0}\right|$. The fake terms in the two solutions are equal up to a relative negative sign, therefore the sum of the terms of Eq. (31) yields the correct complete solution. It is remarkable, however, that there exist fake regions, since in the original equations (28) and (29) there was no indication for that.

As a last remark in this section we emphasize the importance of the cut contributions with a simple example. Let us consider the evolution of $\int d^{3} \mathbf{x}\left\langle\varphi^{2}\right\rangle\left(\mathbf{x}, k_{0}\right)=\left\langle\varphi^{2}\right\rangle\left(\mathbf{k}=\mathbf{0}, k_{0}\right)$ in a free system, without any background field, but with specified initial conditions. The relevant equations are the following [cf. Eqs. (28) and (29)]: 


$$
\begin{gathered}
2 q_{0} k_{0} \bar{G}_{r r}\left(Q, k_{0}\right)=0, \\
\left(Q^{2}+\frac{k_{0}^{2}}{4}-m^{2}\right) \bar{G}_{r r}(Q, K)=0 .
\end{gathered}
$$

Solving the first equation, we would conclude that $\bar{G}_{r r}(Q, t)$ is constant, and thus $\left\langle\varphi^{2}\right\rangle(\mathbf{k}=0, t)$ is constant, too. This would mean that $\left\langle\varphi^{2}\right\rangle(\mathbf{k}=\mathbf{0}, t)$ is a conserved quantity, which it is not, in reality. If we solve the second equation with the first one as a constraint (it yields $q_{0}=0$ ), we find (cf. [11,12]) power-law time dependence, that is, $\left\langle\varphi^{2}\right\rangle(\mathbf{k}$ $=\mathbf{0}, t)$ goes to zero, with some power of time. This again shows that the Boltzmann equation, alone, cannot provide the complete treatment even in the simplest case.

\section{Lessons of the one-loop case}

If we compare the formulas of Eq. (31) to the ones coming from direct perturbation theory [see Eqs. (16) and (17)] we can see that the difference is that in the exact formula there stands $\operatorname{sgn}\left(q_{0} \pm k_{0} / 2\right)$, which is substituted by $\operatorname{sgn}\left(k_{0}\right)$ and $\operatorname{sgn}\left(q_{0}\right)$ in the first and the second form of the above equations, respectively. The reason for this is that when we take, for example, the difference of the two SD equations, $K^{2}$ disappears, and so the corresponding $i k_{0} \varepsilon$ will be missing in the later calculations, too. Therefore, we implicitly assume that the $2 i q_{0} \varepsilon$ term (coming from $2 Q K$ ) dominates it, i.e., $2\left|q_{0}\right|>\left|k_{0}\right|$. This implicit assumption prevents the final result from being applicable everywhere. On the other hand, in the sum equation $2 Q K$ is missing, therefore the corresponding $2 i q_{0} \varepsilon$ will be absent. This, implicitly, requires $2\left|q_{0}\right|$ $<\left|k_{0}\right|$.

Since these considerations concern the left-hand side of the equation, they seem to generalize to the nonperturbative, nonlinear $\mathrm{KB}$ equations as well. Usually one considers the $\mathrm{KB}$ equations as a differential equation [Eq. (28), the Boltzmann equation] and, assuming $K \ll Q$, a constraint (28). According to the previous analysis, this strategy can be applied only for $2\left|q_{0}\right|>\left|k_{0}\right|$, while in the $2\left|q_{0}\right|<\left|k_{0}\right|$ case the opposite strategy, i.e., considering the sum equation (28) as a differential equation and the difference equation (29) as a constraint, seems to be applicable. To cover the full range of $q_{0}$ and $k_{0}$, we have to take the sum of the two solutions.

This strategy preserves the solutions of the usual Boltzmann equations (although with a limited validity range), but provides supplementary terms in the final solution. These correspond to the normal cut regime in the one-loop perturbative case, thus they can describe-especially in the zero mass case-potentially important physics.

\section{HIGHER LOOPS AND PINCH SINGULARITIES}

Higher loops are, of course, suppressed by powers of the coupling constant; therefore, they become important only if the one-loop result is zero or there are IR divergences. The imaginary part of the one-loop result, as we have seen, is zero if $0<K^{2}<4 m^{2}$; at two loops, however, this restriction is relaxed and a nonzero imaginary part is obtained for all $K$. As far as the IR divergences are concerned, in our case the pinch singularities $[7,8]$ are the primary sources of them.

If the product of a retarded and an advanced propagator appears in some objects with the same momentum, then the poles of the propagators pinch the real axis as $\varepsilon \rightarrow 0$ and yield a nonregularizable double pole. If the two momenta are not equal, we expect a singular behavior of the object as the difference of the momenta vanishes. Using the fact that $G_{R} G_{R}$ is not singular in this limit and that $i\left(G_{R}-G_{A}\right)=\varrho$, we can write

$$
\begin{aligned}
G_{R}\left(Q+\frac{K}{2}\right) G_{A}\left(Q-\frac{K}{2}\right)= & G_{R}\left(Q+\frac{K}{2}\right) i \varrho\left(Q-\frac{K}{2}\right) \\
& \text { - nonsingular. }
\end{aligned}
$$

If $K \rightarrow 0$, the free spectral function $\varrho(Q) \sim \delta\left(Q^{2}-m^{2}\right)$ puts $G_{R}$ on-shell: this is the core effect of the pinch singularities. On the other hand, $G_{r r} \sim \varrho$, thus similar singularities appear when we multiply $G_{R, A}$ and $G_{r r}$. We have to treat this case in complete analogy with the $G_{R} G_{A}$ product.

Pinch singularities appear in all higher-order diagrams containing two-particle intermediate states [9]; to see the net effect, we have to sum up all the diagrams of this type. In the linear-response case, these diagrams form ladders.

Which are the kinematic ranges where we have to count with pinch singularities? As we have seen in the Introduction, we can write

$$
\begin{aligned}
2 i \varrho\left(Q-\frac{K}{2}\right) G_{R}\left(Q+\frac{K}{2}\right)= & i \varrho\left(Q-\frac{K}{2}\right)\left(\frac{\Theta\left(\left|2 q_{0}\right|-\left|k_{0}\right|\right)}{Q K}\right. \\
& \left.+\frac{\Theta\left(\left|k_{0}\right|-\left|2 q_{0}\right|\right)}{Q^{2}+K^{2} / 4-m^{2}}\right)\left.\right|_{k_{0} \rightarrow k_{0}+i \varepsilon}
\end{aligned}
$$

There are, therefore, two sensitive regimes: the vanishing $2 Q K$ or the vanishing $Q^{2}+K^{2} / 4-m^{2}$ regime. The presence of $\varrho[Q-(K / 2)]$ in the formula means that these terms are equal when $\varepsilon=0$; because of Landau prescription, however, they represent different kinematical regions when they appear in the denominator.

To resum the pinch singular diagrams, we can use any valid equation where the left-hand side is proportional to $2 Q K$ or $Q^{2}+K^{2} / 4-m^{2}$, respectively, and the right-hand side is nonvanishing in the $2 Q K=0$ or $Q^{2}+K^{2} / 4-m^{2}=0$ limit [9]; the difference in the exact choice of the equation just gives a subleading effect. In linear-response theory, we need a linearized right-hand side. Exactly of this form are the linearized SD equations (A14) of Appendix A when we take sums and differences of pairs of equations. Starting from it, and linearizing around equilibrium in the form

$$
\begin{aligned}
& \bar{G}(Q, K)=G^{\mathrm{eq}}(Q) \bar{\delta}(K)+g(Q, K), \\
& \bar{\Pi}(Q, K)=\Pi^{\mathrm{eq}}(Q) \bar{\delta}(K)+\pi(Q, K),
\end{aligned}
$$

one finds the following equations for the deviations: 


$$
\begin{gathered}
\left(Q^{2}+\frac{K^{2}}{4}-m^{2}\right) 2 g_{r r}=\pi_{a a}\left(G_{a r}^{-}+G_{r a}^{+}\right)+\Pi_{a a}^{+} g_{a r} \\
+\Pi_{a a}^{-} g_{r a}+\pi_{a r} G_{r r}^{-}+\pi_{r a} G_{r r}^{+} \\
+\left(\Pi_{a r}^{+}+\Pi_{r a}^{-}\right) g_{r r}, \\
2 Q K g_{r r}=\pi_{a a}\left(G_{a r}^{-}-G_{r a}^{+}\right)+\Pi_{a a}^{+} g_{a r}-\Pi_{a a}^{-} g_{r a}+\pi_{a r} G_{r r}^{-} \\
-\pi_{r a} G_{r r}^{+}+\left(\Pi_{a r}^{+}-\Pi_{r a}^{-}\right) g_{r r}, \\
\left(Q^{2}+\frac{K^{2}}{4}-m^{2}\right) 2 g_{r a}=\pi_{a r}\left(G_{r a}^{-}+G_{r a}^{+}\right)+\left(\Pi_{a r}^{+}+\Pi_{a r}^{-}\right) g_{r a}, \\
2 Q K g_{r a}=\pi_{a r}\left(G_{r a}^{-}-G_{r a}^{+}\right)+\left(\Pi_{a r}^{+}-\Pi_{a r}^{-}\right) g_{r a},
\end{gathered}
$$

where we have omitted the $(Q, K)$ arguments of the functions and we have introduced the notations $F^{ \pm} \equiv F^{\mathrm{eq}}(Q$ $\pm K / 2)$. The $\mathcal{P}$ dependence is hidden in $\pi_{r a}$ and $\pi_{a r}$ :

$$
\pi_{r a} \rightarrow \mathcal{P}+\pi_{r a}, \quad \pi_{a r} \rightarrow \mathcal{P}+\pi_{a r} .
$$

The self-energies (apart from the term containing $\mathcal{P}$ ) come from loop diagrams, i.e., they consist of propagator products supplemented with some integration. In a linear response, all propagators but one are in equilibrium, so they are functions of space-time coordinate difference. Therefore, the general structure reads, in Fourier space,

$$
\pi(p, q) \sim \int d k d \ell \mathcal{K}(p,-q, k,-\ell) g(k, \ell),
$$

where we divided the momenta into incoming $(p, k)$ and outgoing $(q, \ell)$ ones, but this is just a convention in the signs. Since $\mathcal{K}$ contains equilibrium propagators only, the momentum is conserved, and we can write

$$
\pi(p, q) \sim \int d \ell \mathcal{K}^{\prime}(p,-q, \ell) g(p-q+\ell, \ell) .
$$

The Wigner-transformed function reads

$$
\begin{aligned}
\bar{\pi}(Q, K) & \sim \int d \ell \overline{\mathcal{K}}^{\prime}(Q, K, \ell) \bar{g}(K+\ell, K) \\
& =\int d \ell \overline{\mathcal{K}}^{\prime \prime}(Q, K, \ell) \bar{g}(\ell, K) .
\end{aligned}
$$

In general, we have to sum over the R/A indexes, too. From this form we see that the linearized SD equations are diagonal in $K$.

\section{A. The Boltzmann equations}

As we have argued earlier, the members of the set of SD equations can be differential equations or constraints, according to the relations between $\left|k_{0}\right|$ and $\left|q_{0}\right|$. In the Boltzmann region $2\left|q_{0}\right|>\left|k_{0}\right|$, the differential equations are the second and fourth of Eq. (36); the constraint equations are the first and third ones. We concentrate on the pinch singular regime, which means we assume that $Q K$ is small.
First we examine the equations for $g_{r a}$,

$$
2 Q K g_{r a}=\pi_{a r}\left(G_{r a}^{-}-G_{r a}^{+}\right)+\left(\Pi_{a r}^{+}-\Pi_{a r}^{-}\right) g_{r a} .
$$

If $2\left|q_{0}\right|>\left|k_{0}\right|$ and in the small $Q K$ case the following approximation is valid:

$$
\begin{aligned}
G_{r a}^{ \pm} & =\frac{1}{Q^{2}+K^{2} / 4-m^{2} \pm Q K+i q_{0} \varepsilon} \\
& =\frac{1}{Q^{2}+K^{2} / 4-m^{2}+i q_{0} \varepsilon}+O(Q K),
\end{aligned}
$$

therefore the difference of these functions is zero up to $O(Q K)$. Then the equation for $g_{r a}$ is homogeneous (no mixing with $g_{r r}$ ), therefore a valid ansatz is to assume that $g_{r a}$ $\equiv 0$. In a similar way, we can assume that $g_{a r} \equiv 0$. This is a generally accepted approximation: the usual Boltzmann equation never leads to nonzero $g_{r a}$ and $g_{a r}$.

Having said this, the equation for $g_{r r}$ reads

$$
\begin{aligned}
2 Q K g_{r r}= & \pi_{a a}\left(G_{a r}^{-}-G_{r a}^{+}\right)+\pi_{a r} G_{r r}^{-}-\pi_{r a} G_{r r}^{+} \\
& +\left(\Pi_{a r}^{+}-\Pi_{r a}^{-}\right) g_{r r} .
\end{aligned}
$$

We approximate the free $G_{r r}$ for small $2 Q K$ and $2\left|q_{0}\right|$ $>\left|k_{0}\right|$ as $\left[\right.$ here $\left.n_{ \pm}=n\left(q_{0} \pm k_{0} / 2\right)\right]$

$$
\begin{aligned}
i G_{r r}^{ \pm} & =2 \pi \operatorname{sgn}\left(q_{0} \pm \frac{k_{0}}{2}\right)\left(\frac{1}{2}+n_{ \pm}\right) \delta\left(Q^{2}+\frac{K^{2}}{4}-m^{2} \pm Q K\right) \\
& \approx 2 \pi \operatorname{sgn}\left(q_{0}\right)\left(\frac{1}{2}+n_{ \pm}\right) \delta\left(Q^{2}+\frac{K^{2}}{4}-m^{2}\right)
\end{aligned}
$$

while the difference between $G_{r a}$ and $G_{a r}$, as we have seen in Eq. (42), reads as

$$
G_{a r}^{-}-G_{r a}^{+}=2 \pi i \operatorname{sgn}\left(q_{0}\right) \delta\left(Q^{2}+\frac{K^{2}}{4}-m^{2}\right)+O(Q K) .
$$

Therefore, all explicit terms in the equation are proportional to $\operatorname{sgn}\left(q_{0}\right) \delta\left(Q^{2}+K^{2} / 4-m^{2}\right)$; thus we can have a solution of the form

$$
i g_{r r}(Q, K)=2 \pi \operatorname{sgn}\left(q_{0}\right) \delta\left(Q^{2}+\frac{K^{2}}{4}-m^{2}\right) \delta n(Q, K),
$$

which is consistent with the constraint equations if we omit the right-hand sides (which represent higher loop effects).

If we are interested in the small $\mathbf{k}$ limit of the result, we can make other simplifications as well. Since in this region $K^{2}<0$, it also means a small $k_{0}$, and we can thus neglect $K^{2}$ on the right-hand side. We find finally

$$
\begin{aligned}
2 Q K \delta n= & -\pi_{a a}+\left(\pi_{a r}-\pi_{r a}\right)\left(\frac{1}{2}+n\left(q_{0}\right)\right) \\
& +\left(\Pi_{a r}^{0}-\Pi_{r a}^{0}\right) \delta n .
\end{aligned}
$$


This is the linearized Boltzmann equation when we expand $\pi$ 's in terms of $\delta n$.

In our concrete model (4), using Eq. (46), we find

$$
\begin{aligned}
\pi_{a a}= & -i h^{2} \int d L \varrho(L) \varrho(Q-L)\left[\left(\frac{1}{2}+n\left(q_{0}-\ell_{0}\right)\right) \delta n\left(\ell_{0}\right)\right. \\
& \left.+\left(\frac{1}{2}+n\left(\ell_{0}\right)\right) \delta n\left(q_{0}-\ell_{0}\right)\right] \pi_{a r}-\pi_{r a} \\
= & -i h^{2} \int d L \varrho(L) \varrho(Q-L)\left[\delta n\left(\ell_{0}\right)+\delta n\left(q_{0}-\ell_{0}\right)\right] .
\end{aligned}
$$

The equilibrium value of the self-energy reads

$$
\begin{aligned}
\Pi_{a r}^{0}-\Pi_{r a}^{0}= & -i h^{2} \int d L \varrho(L) \varrho(Q-L) \\
& \times\left[1+n\left(\ell_{0}\right)+n\left(q_{0}-\ell_{0}\right)\right],
\end{aligned}
$$

and thus for the integrand on the right-hand side we find

$$
\begin{aligned}
& \delta n\left(\ell_{0}\right)\left[n\left(q_{0}\right)-n\left(q_{0}-\ell_{0}\right)\right]+\delta n\left(q_{0}-\ell_{0}\right)\left[n\left(q_{0}\right)-n\left(\ell_{0}\right)\right] \\
& +\delta n\left(q_{0}\right)\left[1+n\left(\ell_{0}\right)+n\left(q_{0}-\ell_{0}\right)\right],
\end{aligned}
$$

while the complete equation is

$$
\begin{aligned}
2 Q K \delta n= & -i h^{2} \int d L \varrho(L) \varrho(Q-L) \delta\left(\left[1+n\left(\ell_{0}\right)\right]\right. \\
& \times\left[1+n\left(q_{0}-\ell_{0}\right)\right] n\left(q_{0}\right) \\
& \left.-n\left(\ell_{0}\right) n\left(q_{0}-\ell_{0}\right)\left[1+n\left(q_{0}\right)\right]\right) .
\end{aligned}
$$

This is, indeed, a linearized Boltzmann equation.

This expression is actually zero, since $Q^{2}=m^{2}, L^{2}=m^{2}$, and $(Q-L)^{2}=m^{2}$ cannot be true at the same time. In our model, therefore, there is no one-loop correction to the Boltzmann equation. To get a nonzero right-hand side, we can consider a model with different masses where $m_{1}>m_{2 m m}$ $+m_{3}$ is true, or we go to higher loops in the present model.

\section{B. The normal cut region}

Let us now repeat the same analysis for the cut region, where $\left|k_{0}\right|>\left|2 q_{0}\right|$, the first and the third equations of Eq. (36) are differential equations, while the second and fourth ones are constraints. As before, we concentrate again on the pinch singular regime where $Q^{2}+K^{2} / 4-m^{2}$ is small.

First, we take the equation for $g_{r a}$,

$$
\left(Q^{2}+\frac{K^{2}}{4}-m^{2}\right) 2 g_{r a}=\pi_{a r}\left(G_{r a}^{-}+G_{r a}^{+}\right)+\left(\Pi_{a r}^{+}+\Pi_{a r}^{-}\right) g_{r a} .
$$

In this region the following approximation is valid:

$$
\begin{aligned}
G_{r a}^{ \pm} & =\frac{1}{Q^{2}+K^{2} / 4-m^{2} \pm Q K \pm i k_{0} \varepsilon} \\
& =\frac{ \pm 1}{Q K+i k_{0} \varepsilon}+O\left(Q^{2}+\frac{K^{2}}{4}-m^{2}\right),
\end{aligned}
$$

and so the sum $G_{r a}^{-}+G_{r a}^{+} \approx 0$. The equation is approximately homogeneous in $g_{r a}$; we can therefore assume, as before, that $g_{r a}=g_{a r}=0$.

Then the equation for $g_{r r}$ reads

$$
\begin{aligned}
\left(Q^{2}+\frac{K^{2}}{4}-m^{2}\right) 2 g_{r r}= & \pi_{a a}\left(G_{a r}^{-}+G_{r a}^{+}\right)+\pi_{a r} G_{r r}^{-}+\pi_{r a} G_{r r}^{+} \\
& +\left(\Pi_{a r}^{+}+\Pi_{r a}^{-}\right) g_{r r}
\end{aligned}
$$

We now approximate $G_{r r}$ for small $Q^{2}+K^{2} / 4-m^{2}$ and for $\left|k_{0}\right|>\left|2 q_{0}\right|$ as

$$
\begin{aligned}
i G_{r r}^{ \pm}= & 2 \pi \operatorname{sgn}\left(q_{0} \pm \frac{k_{0}}{2}\right)\left[\frac{1}{2}+n\left(q_{0} \pm \frac{k_{0}}{2}\right)\right] \\
& \times \delta\left(Q^{2}+\frac{K^{2}}{4}-m^{2} \pm Q K\right) \\
\approx & 2 \pi \operatorname{sgn}\left(k_{0}\right)\left[\frac{1}{2}+n\left(\frac{k_{0}}{2} \pm q_{0}\right)\right] \delta(Q K)
\end{aligned}
$$

and, according to the previous approximation,

$$
\begin{aligned}
G_{a r}^{-}+G_{r a}^{+} & \approx \frac{-1}{Q K-i k_{0} \varepsilon}+\frac{1}{Q K+i k_{0} \varepsilon} \\
& =-2 \pi i \operatorname{sgn}\left(k_{0}\right) \delta(Q K) .
\end{aligned}
$$

So finally all known terms are explicitly proportional to $\operatorname{sgn}\left(k_{0}\right) \delta(Q K)$, and we can have, similarly to the Boltzmann case, a solution of the form

$$
i g_{r r}(Q, K)=2 \pi \operatorname{sgn}\left(k_{0}\right) \delta(Q K) f(Q, K),
$$

which satisfies the corresponding constraint equation, omitting again the right-hand side.

In the small $\mathbf{k}$ region $\delta(Q K) \approx 1 /\left|k_{0}\right| \delta\left(q_{0}\right)$, we can therefore substitute $q_{0} \approx 0$. Then the only nonzero momentum components are $k_{0}$ and $\mathbf{q}$, and we collect them in a single four-vector $S=\left(k_{0} / 2, \mathbf{q}\right)$. Using spatial rotation symmetry, $\Pi_{r a}(Q-K / 2)=\Pi_{a r}(S)$, and we can write

$$
\left(S^{2}-m^{2}\right) 2 f=\pi_{a a}+\left(\pi_{a r}+\pi_{r a}\right)\left(\frac{1}{2}+n\left(s_{0}\right)\right)+2 \Pi_{a r}(S) f .
$$

This is a linear but nonlocal wave equation for $f$. On the right-hand side, we have to use the fact that $Q^{2}+K^{2} / 4-m^{2}$ is small, i.e., $S^{2} \approx m^{2}$. 
In our concrete model, at one-loop order $\pi_{a a}\left(k_{0} / 2, \mathbf{q}\right)$ $=\operatorname{Im} \Pi_{a r}(S)=0$ at $S^{2}=m^{2}$. The second equality was proved before. In order to prove the first one, we notice that, using Eq. (57),

$$
\begin{aligned}
\pi_{a a}(Q, K)= & -2 i h^{2} \int d L \varrho(Q-L)\left(\frac{1}{2}+n\left(q_{0}-\ell_{0}\right)\right) \\
& \times \operatorname{sgn}\left(k_{0}\right) 2 \pi \delta(L K) f(L, K) .
\end{aligned}
$$

When $\mathbf{k}=q_{0}=0$, then $\delta(2 L K) \sim \delta\left(\ell_{0}\right)$, and thus $\varrho(Q-L)$ would force $Q-L$ on the mass shell with $q_{0}-\ell_{0}=0$, which is impossible. Thus, at the one-loop order, what remains in Eq. (58) (writing the $\mathcal{P}$ dependence explicitly) is

$$
\begin{aligned}
\left(S^{2}-m^{2}\right) f= & \Pi_{a r}^{0}(S) f-\left(\frac{1}{2}+n\left(s_{0}\right)\right) \frac{h^{2}}{s_{0}} \\
& \times \int \frac{d^{3} \ell}{(2 \pi)^{3}} \frac{1}{(\mathbf{s}-\ell)^{2}+m^{2}} f\left(\ell, s_{0}\right) \\
& +\left(\frac{1}{2}+n\left(s_{0}\right)\right) \mathcal{P}\left(2 s_{0}\right) .
\end{aligned}
$$

We see that the treatment of the two regimes (the Boltzmann and the normal cut regions) is quite symmetric; the only difference comes from whether we consider the sum or the difference of the SD equations as the relevant dynamical differential equation, while treating the other equation as a constraint.

\section{Relaxation time approximation}

The relaxation time approximation for Boltzmann equations is well known. It means that we approximate the righthand side of Eq. (51) as $-2 i q_{0} \Gamma_{B} \delta n$. If this approximation is valid, then $\delta n(t) \sim \exp \left(-\Gamma_{B} t\right)$. In our illustrative model, as we have seen, $\Gamma_{B}=O\left(h^{4}\right)$, since it is zero at the one-loop level.

This approximation scheme can be extended also to the normal cut regime. We assume that the operators acting on the right-hand side of the relevant Eq. (60) can be represented as a diagonal linear operator. After rearrangement on the left-hand side, modified parameters (damping rate and mass) will appear. Then Eq. (60) reads

$$
\left[\left(\frac{k_{0}+i \Gamma_{c}}{2}\right)^{2}-\mathbf{q}^{2}-m_{\text {eff }}^{2}\right] f=\left[\frac{1}{2}+n\left(\frac{k_{0}}{2}\right)\right] \mathcal{P}(K) .
$$

At one-loop order, the imaginary part $\Gamma_{c}=0$, just like in the Boltzmann case, but, in general, it is nonzero at higher loops. Its solution is almost the same as the one-loop level result; only the finite imaginary part requires some care. Equation (57) yields

$$
\begin{aligned}
i g_{r r}(Q, K)= & \frac{2 \pi}{k_{0}} \delta\left(q_{0}\right)\left[\frac{1}{2}+n\left(\frac{k_{0}}{2}\right)\right] \\
& \times \frac{1}{\left(k_{0}+i \Gamma_{c}\right)^{2} / 4-\mathbf{q}^{2}-m_{\mathrm{eff}}^{2}} \mathcal{P}\left(k_{0}\right) .
\end{aligned}
$$

When compared with the definition $i g_{r r}(Q, K)$ $=R(Q, K) \mathcal{P}(K) / 2$, we find for $\int d Q i R$, after evaluating the $q_{0}$ integral,

$$
\begin{aligned}
\int d Q i R(Q, K)= & \frac{1}{\pi^{2} k_{0}}\left[\frac{1}{2}+n\left(\frac{k_{0}}{2}\right)\right] \\
& \times \int_{0}^{\infty} d q \frac{q^{2}}{\left(k_{0}+i \Gamma_{c}\right)^{2} / 4-m_{\mathrm{eff}}^{2}-\mathbf{q}^{2}} .
\end{aligned}
$$

After performing the integration and dropping the infinite piece (mass renormalization), we finally arrive at

$$
\begin{aligned}
\int d Q i R(Q, K)= & \frac{i}{4 \pi k_{0}}\left[1+2 n\left(\frac{k_{0}}{2}\right)\right] \\
& \times \sqrt{\frac{\left(k_{0}+i \Gamma_{c}\right)^{2}}{4}-m_{\mathrm{eff}}^{2}}
\end{aligned}
$$

The discontinuity, calculated as twice the imaginary part, reads

$$
\begin{aligned}
\int \underset{k_{0}}{\operatorname{DisciR}(Q, K)=} & \frac{1}{4 \pi k_{0}}\left[1+2 n\left(\frac{k_{0}}{2}\right)\right] \\
& \times 2 \operatorname{Re} \sqrt{\frac{\left(k_{0}+i \Gamma_{c}\right)^{2}}{4}-m_{\mathrm{eff}}^{2}} \\
= & \frac{1}{4 \pi}\left[1+2 n\left(\frac{k_{0}}{2}\right)\right] \frac{2}{k_{0}} \\
& \times F\left(\frac{k_{0}^{2}}{4}-M^{2}, \frac{k_{0} \Gamma_{c}}{2}\right)
\end{aligned}
$$

where $M^{2}=m_{\mathrm{eff}}^{2}+\Gamma_{c}^{2}$ and

$$
F(x, y)=\frac{1}{\sqrt{2}}\left(x+\sqrt{x^{2}+y^{2}}\right)^{1 / 2} .
$$

Let us first analyze some properties of this result.

(i) If $\Gamma_{c}=0$ then, because of $F(x, y=0)=\Theta(x) \sqrt{x}$, we get back the normal cut part of Eq. (21) with the substitution $m \rightarrow M$.

(ii) Using perturbation theory without resummation, we see IR divergences (coming from higher loops) if $m=0$. This is because $M^{2} \sim h^{2}$, thus we can compute power series in $M^{2}$, which yields 


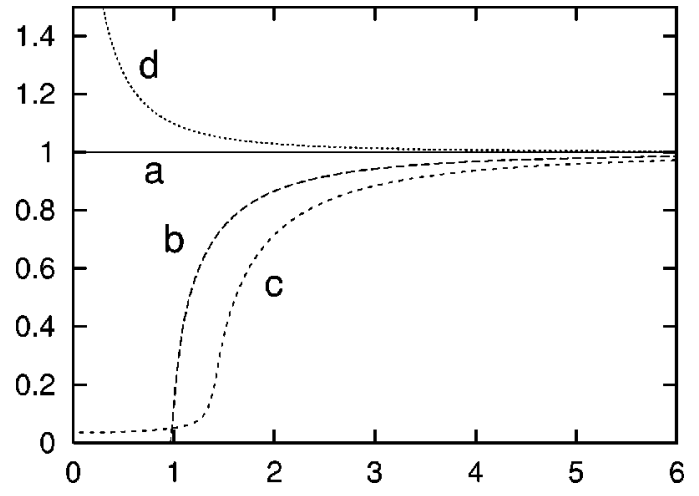

FIG. 1. The function $F / k_{0}$ [cf. Eq. (66)] for different parameters: $a, M=\Gamma_{c}=0 ; b, M=1, \Gamma_{c}=0 ; c, M=1.4, \Gamma_{c}=0.1 ; d, M$ $=0, \Gamma_{c}=0.1$.

$$
\left.\frac{2 F}{k_{0}}\right|_{\text {pert }}=\frac{2}{k_{0}} \sqrt{\frac{k_{0}^{2}}{4}-M^{2}}=1-\frac{2 M^{2}}{k_{0}^{2}}+O\left(h^{4}\right) .
$$

(iii) For $M^{2}=0$,

$$
\left.\frac{2 F}{k_{0}}\right|_{M^{2}=0}=\frac{1}{\sqrt{2}}\left(1+\sqrt{1+\frac{4 \Gamma_{c}^{2}}{k_{0}^{2}}}\right)^{1 / 2} \rightarrow \sqrt{\frac{\Gamma_{c}}{k_{0}}}
$$

yields a divergent (although integrable) behavior.

(iv) At $k_{0}=0, M \neq 0$ we get

$$
\left.\frac{2 F}{k_{0}}\right|_{k_{0} \rightarrow 0}=\frac{\Gamma_{c}}{2 M} \operatorname{sgn}\left(k_{0}\right) .
$$

This is a finite (and nonanalytic) result.

(v) While the free result exhibited a nonanalytic squareroot behavior near the threshold, higher-loop corrections smooth out this nonanalyticity. A nonanalytic behavior implies power-law real time dependence $[11,12]$, which now acquires an exponential damping multiplicative correction, just like the solution of the Boltzmann equation. On the other hand, at $k_{0}=0$, there appears a new nonanalytic contribution.

In order to see these effects, we plotted the function $2 F / k_{0}$ in Fig. 1. Curve $a$ shows the $M=\Gamma_{c}=0$ result; $b$ is the free result with $m=1 ; c$ shows how it changes at higher loops: it acquires a finite shift in the mass as well as a damping that smears out the threshold nonanalyticity (in the plot we have used $M=1.4, \Gamma_{c}=0.1$ ). Finally, curve $d$ shows the $M=0, \quad \Gamma_{c} \neq 0$ case.

\section{COMMENTS ON THE FERMIONIC CASE}

Let us make some comments on the fermionic case. In our simple model, in relaxation time approximation, the only change in the final result, compared to the free case, was a modification just in the threshold function $\Theta(x) \sqrt{x}$ $\rightarrow F(x, y)$, cf. Eq. (65). This seems to be a consequence of the relaxation time approximation, not depending on the specific model, so we may assume that this feature generalizes to other theories, too. In the case of dilepton production, we

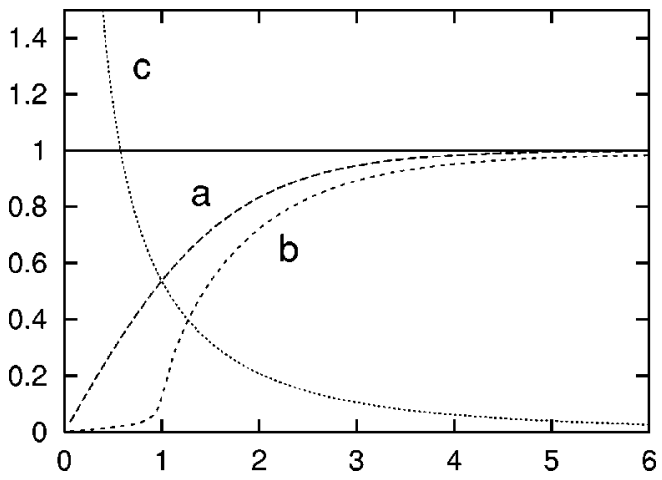

FIG. 2. Expected result of $\left\langle\left[j_{\mu}, j^{\mu}\right]\right\rangle\left(k_{0}\right) / k_{0}^{2}$ for the fermionic case. Curve $a$ is the one-loop result, curve $b$ is the result after resummation. Curve $c$ indicates the size of the two-loop corrections.

have a fermionic system with approximately zero masses. The rate of the dilepton production is proportional to $\left\langle\left[j_{\mu}, j^{\mu}\right]\right\rangle(K)[10]$; when computed perturbatively, this is very similar to our observable $\left\langle\left[\varphi^{2}, \varphi^{2}\right]\right\rangle(K)$ $=\int d Q \operatorname{Disc}_{k_{0}} i R(Q, K)$. At $\mathbf{k}=\mathbf{0}$, the production rate is $\left\langle\left[j_{\mu}, j^{\mu}\right]\right\rangle\left(k_{0}\right) / k_{0}^{2} \sim \tanh \left(\beta k_{0} / 4\right)$; if we assume the same behavior for the higher loop pinch singularities in the fermionic theory as they appear in our model, we obtain changes in the production rate qualitatively similar to Fig. 2.

Here curve $a$ shows the free result and curve $b$ shows the expected result of the resummation, applying the present analysis. Curve $c$ indicates the size of the two-loop perturbative corrections. This figure suggests that, although the twoloop correction is divergent in the $k_{0} \rightarrow 0$ limit, the resummed result can be convergent, or even zero. With the present approximation the discontinuity goes linearly to zero for vanishing momentum, and its slope is $\sim \Gamma_{c} /(4 T M)$. Our approximate result is quite reminiscent of the numerical (MEM method) findings of Karsch et al. [1].

\section{CONCLUSION}

Let us summarize the main results of the paper.

(i) Refinement of the conventional treatment of KadanoffBaym equations. Starting from the pair of nonequilibrium Schwinger-Dyson equations, applying Wigner transformation, and taking their sum and difference, we arrive at the KB equations. The conventional interpretation is the following: for mild variations of the average coordinates, the difference of the SD equations is understood as a conventional firstorder partial differential equation: the quantum Boltzmann equation. In the other (sum) equation, one neglects the dependence on the average coordinates, as a consequence of which it becomes a constraint.

Based on the thorough analysis of the one-loop contribution, and resumming the IR (pinch) singularities arising from higher loops, we tried to argue in the paper that the conventional strategy yields only a partial solution, valid in a restricted kinematical range. In the complementer range we should treat the sum of the SD equations as differential equations while the difference equations play the role of the constraint. 
(ii) Features of the new contribution. The new contribution is responsible at tree level for the appearance of the normal cut; it is IR (pinch) singular at higher loops. We have constructed the relevant equation near the IR-singular regime [see Eq. (58)]. It is a wave equation for the quantity that is the analogue of the particle number variation in the Boltzmann case.

(iii) Solution in the relaxation time approximation. We can try to diagonalize the linear differential equations and keep the eigenmode with the smallest damping rate: this is the relaxation time approximation in effect. In the Boltzmann regime this procedure changes the infinitesimal $i \varepsilon$ term of the Landau prescription to the finite imaginary part $i \Gamma_{B}$, associated with the presence of an effective term in the equation of motion, which is linear in the time derivative. In the normal cut regime there are two contributions: a real one, which is in fact a mass shift, and an imaginary one, which is analogous to the damping rate in the Boltzmann case. Computing the normal cut contribution with this approximation we can observe two effects: the shift of the threshold location and the smoothing of the threshold nonanalyticity. If treated in finite orders of perturbation theory, both effects may lead to divergences.

(iv) Prospects for generalization. The present computation was performed for the case of the cubic scalar theory, and direct computations in other more relevant models are required before one can have quantitative predictions: this is a project for the future. Still, some findings of the present calculation seem to be robust enough to conjecture similar features to be valid in other cases as well. The "dual" interpretation of the KB equations, the wave equation form of the equation describing the cut contributions and the imaginary and real parts in the relaxation time approximation probably generalize to the fermionic case as well. Then we conjecture for the quantity $\left\langle\left[j_{\mu}, j^{\mu}\right]\right\rangle\left(k_{0}\right) / k_{0}^{2}$, relevant to the determination of the dilepton production rate, the behavior indicated in Fig. 2. While two-loop order perturbation theory gives a divergent contribution (curve $c$ ) at threshold, the resummed result shows a much milder behavior (curve $b$ ). This qualitative prediction displays a remarkable similarity to recent numerical data [1].

\section{ACKNOWLEDGMENT}

The author would like to thank to D. Litim, A. Patkós, and P. Petreczky for discussions and proposals. This work was partially supported by the Hungarian Science Fund (OTKA).

\section{APPENDIX A: THE FORMALISM}

In the Keldysh formalism for a complex scalar field, ${ }^{1}$ we define the propagators

$$
\begin{aligned}
i G_{11}(x, y)= & \Theta\left(x_{0}-y_{0}\right)\left\langle\Phi(x) \Phi^{\dagger}(y)\right\rangle \\
& +\Theta\left(y_{0}-x_{0}\right)\left\langle\Phi^{\dagger}(y) \Phi(x)\right\rangle,
\end{aligned}
$$

\footnotetext{
${ }^{1}$ For fermionic fields the formalism is analogous, except for some appropriately placed minus signs; see [7].
}

$$
\begin{gathered}
i G_{12}(x, y)=\left\langle\Phi^{\dagger}(y) \Phi(x)\right\rangle, \\
i G_{22}(x, y)=\Theta\left(y_{0}-x_{0}\right)\left\langle\Phi(x) \Phi^{\dagger}(y)\right\rangle \\
+\Theta\left(x_{0}-y_{0}\right)\left\langle\Phi^{\dagger}(y) \Phi(x)\right\rangle, \\
i G_{21}(x, y)=\left\langle\Phi(x) \Phi^{\dagger}(y)\right\rangle,
\end{gathered}
$$

where the expectation value is defined in general as

$$
\langle\hat{A}\rangle=\operatorname{Tr} \hat{\rho} \hat{A}
$$

where $\hat{\rho}$ is the density matrix. Formally, we introduce two types of fields ( $\Phi_{1}$ and $\left.\Phi_{2}\right)$, which are representations of the original field on the different branches of the closed time contour. Their expectation values are defined as $\left\langle\Phi_{i} \Phi_{j}\right\rangle$ $=i G_{i j}$.

These propagators are not independent. There is a relation between them,

$$
G_{11}+G_{22}=G_{12}+G_{21}
$$

In fact, there are only two independent expectation values, e.g., $G_{12}$ and $G_{21}$, since $G_{11}$ and $G_{22}$ are combinations of these two and the theta function. In equilibrium, even these two are related through the Kubo-Martin-Schwinger (KMS) condition [7].

While these propagators determine the free theory, in case of interactions we have to modify the interaction Lagrangian as (cf. [7])

$$
\mathcal{L}_{I}(\Phi) \rightarrow \mathcal{L}_{I}\left(\Phi_{1}\right)-\mathcal{L}_{I}\left(\Phi_{2}\right)
$$

In the R/A formalism [13], we introduce new fields

$$
\Phi_{r}=\frac{\Phi_{1}+\Phi_{2}}{2}, \quad \Phi_{a}=\Phi_{1}-\Phi_{2} .
$$

Their time-ordered products [denoting $\Phi_{i}(x)$ by $\Phi_{i}$ and $\Phi_{i}^{\dagger}(y)$ by $\left.\Phi_{i}^{\prime}\right]$ are expressible through the two-point functions as follows:

$$
\begin{aligned}
G_{r r} & =\frac{1}{4 i}\left\langle\Phi_{1} \Phi_{1}^{\prime}+\Phi_{1} \Phi_{2}^{\prime}+\Phi_{2} \Phi_{1}^{\prime}+\Phi_{2} \Phi_{2}^{\prime}\right\rangle \\
& =\frac{1}{4}\left(G_{11}+G_{12}+G_{21}+G_{22}\right)=\frac{G_{12}+G_{21}}{2}, \\
G_{r a} & =\frac{1}{2 i}\left\langle\Phi_{1} \Phi_{1}^{\prime}-\Phi_{1} \Phi_{2}^{\prime}+\Phi_{2} \Phi_{1}^{\prime}-\Phi_{2} \Phi_{2}^{\prime}\right\rangle \\
& =\frac{1}{2}\left(G_{11}-G_{12}+G_{21}-G_{22}\right)=G_{11}-G_{12}, \\
G_{a r} & =\frac{1}{2 i}\left\langle\Phi_{1} \Phi_{1}^{\prime}+\Phi_{1} \Phi_{2}^{\prime}-\Phi_{2} \Phi_{1}^{\prime}-\Phi_{2} \Phi_{2}^{\prime}\right\rangle \\
& =\frac{1}{2}\left(G_{11}+G_{12}-G_{21}-G_{22}\right)=G_{11}-G_{21},
\end{aligned}
$$




$$
\begin{aligned}
G_{a a} & =-i\left\langle\Phi_{1} \Phi_{1}^{\prime}-\Phi_{1} \Phi_{2}^{\prime}-\Phi_{2} \Phi_{1}^{\prime}+\Phi_{2} \Phi_{2}^{\prime}\right\rangle \\
& =G_{11}-G_{12}-G_{21}+G_{22}=0 .
\end{aligned}
$$

In a nonequilibrium situation, the propagators depend on the two space-time coordinates separately, and one can define several integral representations for the propagators. The double Fourier transform of a function of two arguments is defined as

$$
\begin{gathered}
f(p, \ell)=\int d x d y e^{i p x-i \ell y} f(x, y), \\
f(x, y)=\int d p d \ell e^{-i p x+i \ell y} f(x, y),
\end{gathered}
$$

where $d p=d^{D} p /(2 \pi)^{D}$ in $D$ dimensions. Note that we define the momentum of the $y$ variable with a relative negative sign. We use the same symbols for the function and its Fourier transform; the type of the argument defines which function we are dealing with. The Wigner transform $\bar{f}$ of a function $f$ with two arguments is defined as

$$
\bar{f}(u, X)=f\left(X+\frac{u}{2}, X-\frac{u}{2}\right), \quad f(x, y)=\bar{f}\left(x-y, \frac{x+y}{2}\right),
$$

i.e., it understands the function to depend on the relative and average coordinates. Fourier transformation with respect to these coordinates yields

$$
\begin{aligned}
\bar{f}(Q, K) & =\int d u d X e^{i Q u+i K X} \bar{f}(u, X) \\
& =\int d x d y e^{i Q(x-y)+i K(x+y) / 2} f(x, y) \\
& =f\left(Q+\frac{K}{2}, Q-\frac{K}{2}\right), \\
& f(p, \ell)=\bar{f}\left(\frac{p+\ell}{2}, p-\ell\right) .
\end{aligned}
$$

The derivatives with respect to $x$ and $y$ transform as

$$
\begin{gathered}
i \partial_{x} f(x, y) \stackrel{\text { Fourier }}{\rightarrow} p f(p, \ell) \stackrel{\text { Wigner }}{\rightarrow}\left(Q+\frac{K}{2}\right) \bar{f}(Q, K), \\
i \partial_{y} f(x, y) \stackrel{\text { Fourier }}{\rightarrow}-\ell f(p, \ell) \stackrel{\text { Wigner }}{\rightarrow}\left(-Q+\frac{K}{2}\right) \bar{f}(Q, K) .
\end{gathered}
$$

Later on we will use the Schwinger-Dyson equations for propagators. It is used to resum bubble series

$$
\begin{aligned}
G_{a b} & =G_{a b}^{0}+G_{a c}^{0} \Pi_{c d} G_{d b}^{0}+\cdots=G_{a b}^{0}+G_{a c}^{0} \Pi_{c d} G_{d b} \\
& =G_{a b}^{0}+G_{a c} \Pi_{c d} G_{d b}^{0} .
\end{aligned}
$$

The indices refer to space-time coordinates as well as to internal indices (e.g., Keldysh indices). To have a differential form we apply the free time evolution kernel on both sides. In the R/A formalism these kernels satisfy "twin" equations, which differ only in the variable to which the derivatives are referring:

$$
\begin{gathered}
\left(\partial_{x, y}^{2}+m^{2}\right) G_{r r}^{0}(x, y)=0, \\
\left(\partial_{x, y}^{2}+m^{2}\right) G_{r a}^{0}(x, y)=\left(\partial_{x, y}^{2}+m^{2}\right) G_{a r}^{0}(x, y) \\
=-\delta(x-y) .
\end{gathered}
$$

Using these, we find the SD equations,

$$
\begin{aligned}
& -\left(\partial_{x}^{2}+m^{2}\right) G_{r r}=\int d z\left(\Pi_{a a} G_{a r}+\Pi_{a r} G_{r r}\right), \\
& -\left(\partial_{y}^{2}+m^{2}\right) G_{r r}=\int d z\left(G_{r a} \Pi_{a a}+G_{r r} \Pi_{r a}\right) \\
& -\left(\partial_{x}^{2}+m^{2}\right) G_{r a}=\delta(x-y)+\int d z \Pi_{a r} G_{r a}, \\
& -\left(\partial_{y}^{2}+m^{2}\right) G_{r a}=\delta(x-y)+\int d z G_{r a} \Pi_{a r}, \\
& -\left(\partial_{x}^{2}+m^{2}\right) G_{a r}=\delta(x-y)+\int d z \Pi_{r a} G_{a r}, \\
& -\left(\partial_{y}^{2}+m^{2}\right) G_{a r}=\delta(x-y)+\int d z G_{a r} \Pi_{r a} .
\end{aligned}
$$

Here we have used the fact that $G_{a a}=0$ is consistent with SD equations only if $\Pi_{r r}=0$. After the Wigner-Fourier transformations, Eqs. (A13) look a little more complicated,

$$
\begin{aligned}
& {\left[\left(Q+\frac{K}{2}\right)^{2}-m^{2}\right] \bar{G}_{r r}(Q, K)} \\
& =\int d k\left[\bar{\Pi}_{a a}\left(Q+\frac{k}{2}, K-k\right) \bar{G}_{a r}\left(Q-\frac{K-k}{2}, k\right)\right. \\
& \left.\quad+\Pi_{a r}\left(Q+\frac{k}{2}, K-k\right) \bar{G}_{r r}\left(Q-\frac{K-k}{2}, k\right)\right], \\
& {\left[\left(Q-\frac{K}{2}\right)^{2}-m^{2}\right] \bar{G}_{r r}(Q, K)} \\
& =\int d k\left[\bar{\Pi}_{a a}\left(Q-\frac{k}{2}, K-k\right) \bar{G}_{r a}\left(Q+\frac{K-k}{2}, k\right)\right. \\
& \left.\quad+\bar{\Pi}_{r a}\left(Q-\frac{k}{2}, K-k\right) \bar{G}_{r r}\left(Q+\frac{K-k}{2}, k\right)\right],
\end{aligned}
$$




$$
\begin{array}{rlrl}
{\left[\left(Q+\frac{K}{2}\right)^{2}-m^{2}\right] \bar{G}_{r a}(Q, K)} & D & =\delta\left(2 q_{0} k_{0}-2 q k x\right) \delta\left(q_{0}^{2}-q^{2}+\frac{K^{2}}{4}-m^{2}\right) \\
= & \bar{\delta}(K)+\int d k \bar{\Pi}_{a r}\left(Q+\frac{k}{2}, K-k\right) & & \frac{1}{2\left|k_{0}\right|} \delta\left(q_{0}-\frac{q k x}{k_{0}}\right) \delta\left(q^{2}\left(\frac{k^{2} x^{2}}{k_{0}^{2}}-1\right)+\frac{K^{2}}{4}-m^{2}\right) \\
& \times \bar{G}_{r a}\left(Q-\frac{K-k}{2}, k\right), & & \frac{\left|k_{0}\right|}{2} \frac{1}{\left|k_{0}^{2}-k^{2} x^{2}\right|} \delta\left(q_{0}-\frac{q k x}{k_{0}}\right) \delta\left(q^{2}-\frac{k_{0}^{2}}{4} \frac{K^{2}-4 m^{2}}{k_{0}^{2}-k^{2} x^{2}}\right) \\
{\left[\left(Q-\frac{K}{2}\right)^{2}-m^{2}\right] \bar{G}_{r a}(Q, K)} & & \frac{\left|k_{0}\right|}{2} \frac{1}{\left|k_{0}^{2}-k^{2} x^{2}\right|} \delta\left(q_{0}-\frac{q k x}{k_{0}}\right) \frac{1}{2 q} \\
= & \bar{\delta}(K)+\int d k \Pi_{a r}\left(Q-\frac{k}{2}, K-k\right) & & \times\left[\delta\left(q-\frac{k_{0}}{2} \mathcal{Z}\right)+\delta\left(q+\frac{k_{0}}{2} \mathcal{Z}\right)\right] \\
& \times \bar{G}_{r a}\left(Q+\frac{K-k}{2}, k\right), & \frac{1}{2 \mathcal{Z}\left|k_{0}^{2}-k^{2} x^{2}\right|} \delta\left(q_{0}-\frac{k x}{2} \operatorname{sgn}\left(k_{0}\right) \mathcal{Z}\right) \delta\left(q-\frac{\left|k_{0}\right|}{2} \mathcal{Z}\right) .
\end{array}
$$

$$
\begin{aligned}
& {\left[\left(Q+\frac{K}{2}\right)^{2}-m^{2}\right] \bar{G}_{a r}(Q, K) } \\
&= \bar{\delta}(K)+\int d k \bar{\Pi}_{r a}\left(Q+\frac{k}{2}, K-k\right) \\
& \times \bar{G}_{a r}\left(Q-\frac{K-k}{2}, k\right), \\
& {[(Q-}\left.\left.\frac{K}{2}\right)^{2}-m^{2}\right] \bar{G}_{a r}(Q, K) \\
&= \bar{\delta}(K)+\int d k \bar{\Pi}_{r a}\left(Q-\frac{k}{2}, K-k\right) \\
& \quad \times \bar{G}_{a r}\left(Q+\frac{K-k}{2}, k\right),
\end{aligned}
$$

where $\bar{\delta}(p)=(2 \pi)^{D} \delta(p)$ in $D$ dimensions. The $\pm K$ equations are related to each other by complex conjugation and $K \rightarrow-K$ substitution.

\section{APPENDIX B: DETAILED COMPUTATION OF SEC. III}

In the product of $\delta$ functions (17), we observe that the first delta function $\delta\left(Q^{2}+Q K+K^{2} / 4-m^{2}\right)$ implies $Q K$ $=m^{2}-Q^{2}-K^{2} / 4$, which yields $\delta\left(Q^{2}-Q K+K^{2} / 4-m^{2}\right)$ $\rightarrow \delta(2 Q K)$. This, in turn, means that one can set $Q K=0$ in its coefficient, and therefore we can write $\delta\left(Q^{2}+Q K\right.$ $\left.+K^{2} / 4-m^{2}\right) \rightarrow \delta\left(Q^{2}+K^{2} / 4-m^{2}\right)$. We obtain in this way the second form of Eq. (17). In order to get the third form, the following steps are done [we use the notations of Eq. (14)]:

In the third line we have introduced the quantity $\mathcal{Z}$ $=\sqrt{\left(K^{2}-4 m^{2}\right) /\left(k_{0}^{2}-k^{2} x^{2}\right)}$. In order to get the fourth line, we used the fact that, since $q$ is positive, only one of the two $\delta$ functions appearing on the third line can be satisfied, therefore $q=\left|k_{0}\right| \mathcal{Z} / 2$. The expression is nonzero if $\mathcal{Z}$ is real, i.e., when $\left(K^{2}-4 m^{2}\right) /\left(k_{0}^{2}-k^{2} x^{2}\right)>0$. To examine this condition we split the $K^{2}$ range into two regions: (i) $K^{2}>4 m^{2}$; then $k_{0}^{2}>k^{2}>k^{2} x^{2}$ (since $|x|<1$ ), so the numerator and the denominator are positive; (ii) $K^{2}<4 \mathrm{~m}^{2}$; then the numerator is negative, the denominator thus has to be negative as well. This yields $k_{0}^{2}<k^{2} x^{2}$, which implies $1>|x|>\left|k_{0}\right| / k$ and $k^{2}$ $>k_{0}^{2}$, i.e., $K^{2}<0$. Therefore, $\mathcal{Z}$ is real if $K^{2}>4 m^{2}$ or $K^{2}$ $<0$.

We can examine the relative size of $\left|q_{0}\right|$ and $\left|k_{0}\right|$ from the last form of Eq. (17). To find it, we rewrite $\mathcal{Z}$ as

$$
\mathcal{Z}^{2}=\frac{K^{2}-4 m^{2}}{k_{0}^{2}-k^{2} x^{2}}=1-\frac{k^{2}\left(1-x^{2}\right)+4 m^{2}}{k_{0}^{2}-k^{2} x^{2}} .
$$

The numerator is always positive, thus if $K^{2}>4 m^{2}$, then $|x| \leqslant 1, \quad \mathcal{Z} \leqslant 1$, and one has $\left|q_{0}\right|=(|k x| / 2) \mathcal{Z} \leqslant k / 2 \leqslant\left|k_{0}\right| / 2$; if $K^{2}<0$, then $1 \geqslant|x| \geqslant\left|k_{0}\right| / k, \quad \mathcal{Z} \geqslant 1$, giving $\left|q_{0}\right|=(|k x| / 2) \mathcal{Z}$ $\geqslant\left|k_{0}\right| / 2$.

For the $Q$ integration appearing in Eq. (19),

$$
\begin{aligned}
\underset{k_{0}}{\operatorname{DisciR}(Q, K)=} & \frac{4 \pi^{2}}{\sqrt{\left(k_{0}^{2}-k^{2} x^{2}\right)\left(K^{2}-4 m^{2}\right)}} \\
& \times \delta\left(q_{0}-\frac{k x}{2} \operatorname{sgn}\left(k_{0}\right) \mathcal{Z}\right) \delta\left(q-\frac{\left|k_{0}\right|}{2} \mathcal{Z}\right) \\
& \times\left\{\Theta ( K ^ { 2 } - 4 m ^ { 2 } ) \left[1+n\left(\frac{k_{0}}{2}-q_{0}\right)\right.\right. \\
& \left.+n\left(\frac{k_{0}}{2}+q_{0}\right)\right]+\Theta\left(-K^{2}\right)\left[n\left(q_{0}-\frac{k_{0}}{2}\right)\right. \\
& \left.\left.-n\left(q_{0}+\frac{k_{0}}{2}\right)\right]\right\},
\end{aligned}
$$


we write the integration measure as

$$
\frac{1}{8 \pi^{3}} \int d q_{0} d q d x q^{2}
$$

The following change of variable is made:

$$
\begin{gathered}
x \rightarrow y=\frac{k x}{2} \mathcal{Z}, \frac{d y}{d x}=\frac{k_{0}^{2}}{2} \sqrt{\frac{K^{2}-4^{2}}{\left(k_{0}^{2}-k^{2} x^{2}\right)^{3}},} \\
y \in \frac{1}{2} \begin{cases}{\left[-k \mathcal{Z}_{1}, k \mathcal{Z}_{1}\right]} & \text { if } K^{2}>4 m^{2} \\
{\left[-\infty,-k \mathcal{Z}_{1}\right] \cup\left[k \mathcal{Z}_{1}, \infty\right]} & \text { if } K^{2}<0,\end{cases}
\end{gathered}
$$

where $\mathcal{Z}_{1}=\mathcal{Z}(x=1)=\sqrt{1-4 m^{2} / K^{2}}$. Then we have

$$
\begin{aligned}
& \frac{1}{8 \pi^{3}} \int_{-\infty}^{\infty} d q_{0} \int_{0}^{\infty} d q\left[\Theta\left(K^{2}-4^{2}\right) \int_{-k \mathcal{Z}_{1} / 2}^{k \mathcal{Z}_{1} / 2} d y+\Theta\left(-K^{2}\right)\right. \\
& \left.\times \int_{-\infty}^{-k \mathcal{Z}_{1} / 2} d y+\Theta\left(-K^{2}\right) \int_{k \mathcal{Z}_{1} / 2}^{\infty} d y\right] \frac{2 q^{2}}{k_{0}^{2} k} \sqrt{\frac{\left(k_{0}^{2}-k^{2} x^{2}\right)^{3}}{K^{2}-4 m^{2}}} .
\end{aligned}
$$

Putting the ingredients together, we find the result quoted in Eq. (20):

$$
\begin{aligned}
\int \underset{k_{0}}{d Q \operatorname{DisciR}(Q, K)=} & \frac{\operatorname{sgn}\left(k_{0}\right)}{8 \pi k}\left\{\Theta\left(K^{2}-4 m^{2}\right) \int_{-k \mathcal{Z}_{1} / 2}^{k \mathcal{Z}_{1} / 2} d y\right. \\
& \times\left[1+n\left(\frac{\left|k_{0}\right|}{2}-y\right)+n\left(\frac{\left|k_{0}\right|}{2}+y\right)\right] \\
& +2 \Theta\left(-K^{2}\right) \int_{k Z_{1} / 2}^{\infty} d y\left[n\left(y-\frac{\left|k_{0}\right|}{2}\right)\right. \\
& \left.\left.-n\left(y+\frac{\left|k_{0}\right|}{2}\right)\right]\right\}
\end{aligned}
$$

[1] F. Karsch, E. Laermann, P. Petreczky, S. Stickan, and I. Wetzorke, Phys. Lett. B 530, 147 (2002).

[2] T. Altherr and P. Aurenche, Z. Phys. C 45, 99 (1989).

[3] E. Braaten, R. D. Pisarski, and T. Ch. Yuan, Phys. Rev. Lett. 64, 2242 (1990).

[4] P. Aurenche, F. Gelis, and H. Zaraket, Phys. Rev. D 61, 116001 (2000); 62, 096012 (2000).

[5] P. Arnold, G. D. Moore, and L. G. Yaffe, J. High Energy Phys. 11, 057 (2001); 12, 009 (2001); 06, 030 (2002).

[6] D. Boyanovsky, H. J. de Vega, and S.-Y. Wang, Phys. Rev. D 61, 065006 (2000).

[7] N. P. Landsmann and Ch. G. van Weert, Phys. Rep. 145, 141 (1987).
[8] T. Altherr and D. Seibert, Phys. Lett. B 333, 149 (1994).

[9] A. Jakovác, Phys. Rev. D 65, 085029 (2002).

[10] J. Kapusta, Phys. Lett. 136B, 201 (1984); L. D. McLerran and T. Toimela, Phys. Rev. D 31, 545 (1985).

[11] A. Jakovác, A. Patkós, P. Petreczky, and Zs. Szép, Phys. Rev. D 61, 025006 (2000).

[12] D. Boyanovsky, I. D. Lawrie, and D. S. Lee, Phys. Rev. D 54, 4013 (1996); D. Boyanovsky, M. D’Attanasio, H. J. de Vega, and R. Holman, ibid. 54, 1748 (1996).

[13] E. Wang and U. Heinz, Phys. Lett. B 471, 208 (1999); K.-C. Chou, Z.-B. Su, B.-L. Lao, and L. Yu, Phys. Rep. 118, 1 (1985). 